\title{
Congestive Heart Failure Exhibited Higher BMI With Lower Energy Intake and Lower Physical Activity Level: Data From the National Health and Examination Nutrition Survey
}

\section{Tianyu Xu}

Chinese Academy of Medical Sciences \& Peking Union Medical College Fuwai Hospital

Haobing Zhou

Southern Medical University Nanfang Hospital

\section{Zhuang Ma}

Southern Medical University Nanfang Hospital

Hao Zhang

Southern Medical University Nanfang Hospital

\section{Yuli Huang}

Shunde Hospital of Southern Medical University

\section{Qingchun Zeng}

Southern Medical University Nanfang Hospital

\section{Dingli Xu}

Southern Medical University Nanfang Hospital

\section{Yuhui Zhang}

Chinese Academy of Medical Sciences \& Peking Union Medical College Fuwai Hospital

Jian Zhang ( $\square$ fwzhangjian62@126.com )

Chinese Academy of Medical Sciences \& Peking Union Medical College Fuwai Hospital

\section{Research}

Keywords: congestive heart failure, BMI, total nutrient intakes, physical activity, hemodilution, obesity

Posted Date: March 2nd, 2021

DOl: https://doi.org/10.21203/rs.3.rs-268704/v1

License: (1) This work is licensed under a Creative Commons Attribution 4.0 International License. Read Full License 


\section{Abstract}

Introduction: Despite nutritional deficiency existed in congestive heart failure (CHF), there is a large amount of CHF patients suffer from obesity. This study aimed to identify the differences for increased BMI or obesity in CHF patients.

Methods: This cross-sectional study included adults from the National Health and Nutrition Examination Survey 2007-2016. Differences were compared between CHF participants versus non-CHF participants, and $\mathrm{BMI} \geq 30 \mathrm{~kg} / \mathrm{m}^{2}$ versus $\mathrm{BMI}<30 \mathrm{~kg} / \mathrm{m}^{2} \mathrm{CHF}$ participants.

Results: CHF participants were with higher BMI, lower energy and macronutrients intakes, lower physical activity level and longer rest time, lower hematocrit and hemoglobin level (all $P<0.05$ ) than non-CHF participants. The prevalence of $\mathrm{BMI} \geq 30 \mathrm{~kg} / \mathrm{m}^{2}$ in patients with $\mathrm{CHF}$ was $53.48 \%$. There was no significant difference observed in energy, macronutrients intake between CHF patients with $\mathrm{BMI} \geq$ $30 \mathrm{~kg} / \mathrm{m}^{2}$ or $<30 \mathrm{~kg} / \mathrm{m}^{2}$. The water intake $(P=0.032)$, sedentary time $(P=0.002)$, and hematocrit $(P$ $=0.028$ ) were significantly different between $\mathrm{CHF}$ with $\mathrm{BMI} \geq 30 \mathrm{~kg} / \mathrm{m}^{2}$ and with $<30 \mathrm{~kg} / \mathrm{m}^{2}$.

Conclusion: Compared with non-CHF participants, CHF patients exhibited higher BMI with lower energy and macronutrients intakes, lower physical activity level, longer rest time and hemodilution with lower hematocrit and hemoglobin level. Among CHF patients with $\mathrm{BMI} \geq 30 \mathrm{~kg} / \mathrm{m}^{2}$, higher sedentary time and hematocrit were observed.

\section{Introduction}

Congestive heart failure (CHF) is a complex clinical condition that represents the final evolution of all cardiac disease and a global public health problem which affects an estimated 26 million worldwide [1], which has a tremendous economic impact on the public healthcare system [2]. Studies revealed that CHF is associated with alterations in cardiac energy metabolism [3] and that nutrition intake may influence the evolution of the disease progress [4]. It was reported that a deficiency in energy and macronutrient intakes among CHF patients, which caused an undernourished or malnutritional condition [5-7]. Obesity [body mass index $(\mathrm{BMI}) \geq 30 \mathrm{~kg} / \mathrm{m}^{2}$ ], with up to $40 \%$ prevalence among CHF patients [8], is recognized as a major independent risk factor for the development of $\mathrm{CHF}$ [9] and has a paradoxical impact on the prognosis of CHF $[10,11]$. Studies indicated that a nonlinear U-shaped association between $\mathrm{BMI}$ and the risk of HF mortality, with a greater risk from being at the lowest group (mean BMI $=19.43 \mathrm{~kg} / \mathrm{m}^{2}$ ), rather than being at the top category (mean $\mathrm{BMI}=30.16 \mathrm{~kg} / \mathrm{m}^{2}$ ) [12]. The beneficial effects of weight loss in $\mathrm{HF}$ patients are still controversial. Given the view that despite nutritional deficiency existed in CHF, there is a large amount of CHF patients suffer from obesity, and we still lacked studies to explain the reasons for the increased BMI in CHF patients. In this research, we used the data from the National Health and Examination Nutrition Survey (NHANES) 2007-2016 to identify the differences for increased BMI or obesity in CHF patients. 


\section{Method}

\section{Dataset}

The NHANES data, a nationally representative multistage cross-sectional survey of the nonmilitary and noninstitutionalized population of the United States, was used as the data source [13]. The data is released by the National Center for Health Statistics once every 2 years. Each survey cycle collects selfreported and directly measured information from participants who undergo a series of questionnaires in a detailed in-home interview such as health conditions, behaviors and dietary intakes, and a physical examination during mobile examination. Also, each participant provides their blood for laboratory tests.

\section{Study population}

To identify the study population, we implemented the following exclusion criteria: participants $<18$ years old, participants who were pregnant or breastfeeding at the time of the survey, and participants who lack information on CHF diagnosis, dietary data, examination data, laboratory data or questionnaire data. The final sample for this study consisted of $660 \mathrm{CHF}$ participants and 20,923 non-CHF participants.

\section{Definition of CHF and obesity}

CHF was self-reported and was obtained from the medical conditions files that were administered in the home by an interviewer using the Computer-Assisted Personal Interviewing system as part of the survey participant household interview. The final sample provided yes/no responses to the following question: "Has a doctor or other health professional ever told \{you/SP\} that $\{y$ ou/s/he\}... had congestive HF?"

Height and weight were measured at the MEC examination. BMI was calculated as weight in kilograms divided by the square of height in meters. BMI was analyzed as both a continuous variable and as a categorical variable dichotomizing into $\mathrm{BMI} \geq 30 \mathrm{~kg} / \mathrm{m}^{2}$ (obese) and $\mathrm{BMI}<30 \mathrm{~kg} / \mathrm{m}^{2}$ (non-obese) or into four groups: $\mathrm{BMI}<18.5 \mathrm{~kg} / \mathrm{m}^{2}$ (lean), BMI $18.5-24.9 \mathrm{~kg} / \mathrm{m}^{2}$ (normal-weight), BMI 25-29.9 kg/m² (overweight) and $\mathrm{BMI} \geq 30 \mathrm{~kg} / \mathrm{m}^{2}$ (obese) [14].

\section{Sample demographics, dietary condition and other covariates}

We obtained information on age and sex from the NHANES self-reported demographics data. Blood pressures and heart rate were measured in the MEC examination by a physician. Averages of up to 4 values for systolic and diastolic blood pressures were recorded. Intakes of energy, water, and macronutrients, which was consisted of protein, carbohydrate, total sugars, total fat, and cholesterol, were obtained from self-reported dietary data, and an average of two days was recorded. Physical activity, including work, and recreational activities, was classified as vigorous, moderate, bicycling, or walking, and sedentary, and were obtained by self-report the number of days they engaged in that activity in a typical week and the average duration they engaged in that activity. For each activity, the Metabolic Equivalent of Task (MET)-hr-week was calculated by multiplying the number of days, the mean duration and the respective MET level (MET-hr-week = days $*$ duration $*$ MET level). The MET levels for each activity are 
provided as vigorous work/recreational-related activity $=8 \mathrm{MET}$, moderate work/recreational-related activity=4 MET, walking or bicycling for transportation=4 MET. It was analyzed as a continuous variable for total activates MET and MET in each activity. Hematocrit (\%) and hemoglobin ( $\mathrm{g} / \mathrm{dL})$, which may imply hemodilution [15], were acquired from blood collected during the MEC examination using methods described by the US Department of Health and Human Services.

\section{Statistical Analysis}

Data are expressed as the mean \pm standard deviation or as the number (\%). Differences between groups were tested by the chi-square test for categorical variables and independent Student's t-test, ANOVA with Tukey's post-hoc analysis for continuous variables, as appropriate. Since the sample size is large, a simple application of the Lyapunov or Lindeberg's Central Limit Theorem guarantees large sample convergence of the weighted mean to a standard normal distribution, ensuring that the t-statistic would have a limiting t-distribution. A P value $<0.05$ was considered statistically significant. All tests were twosided. All analyses were performed using R: A Language and Environment for Statistical Computing, version 3.1.3 (R Foundation for Statistical Computing, Vienna, Austria).

\section{Results}

\section{Participants' characteristics between CHF and non-CHF}

The flow chart for participant selection is outlined in Fig. 1. Table 1 presents the clinical characteristics of CHF and non-CHF participants in the NHANES 2007-2016. As noted, CHF participants were older than non-CHF participants $(P<0.05)$ with higher systolic blood pressure, lower diastolic blood pressure, and lower heart rate (all $P<0.05)$. Total nutrients intakes, including energy, protein, total carbohydrate, total sugar, total fat, and cholesterol as well as water were much lower compared with non-CHF patients (all $P$ $<0.05)$. Also, CHF participants had lower physical activity level $(P<0.05)$ and longer sedentary time $(P<$ 0.05). Moreover, hemoglobin and hematocrit were lower in CHF participants $(P<0.05)$. However, BMI was higher in CHF patients than counterparts $(P<0.05)$. 
Table 1

Characteristics of Congestive Heart Failure group and non-Congestive Heart Failure participants

\section{Congestive Heart Failure $(n=660)$}

Non-Congestive Heart failure ( $\mathrm{n}$ $=20,923)$
$P$ value

Demographic and Weight Characteristics, Mean (SD)

Age $(y)$

$66.46(12.72)$

$48.65(17.51)$

$<$

0.001

Race

Male (No. ,\%)

$360(54.55)$

$10,153(48.53)$

BMI $\left(\mathrm{kg} / \mathrm{m}^{2}\right)$

$31.96(8.54)$

$28.99(6.80)$

$\mathrm{SBP}(\mathrm{mmHg})$

$129.82(21.94)$

$123.26(18.17)$

0.001

DBP $(\mathrm{mmHg})$

$65.57(15.03)$

$70.03(12.76)$

$<$

0.001

Heart rate (beats/min)

$71.02(12.69)$

$72.81(12.15)$

$<$

0.001

Total Nutrient Intakes, Mean (SD)

Energy (kcal)

$1734.71(717.33)$

$2051.32(872.86)$

$<$

0.001

Protein (gm)

69.85 (31.39)

80.65 (36.92)

0.001

Carbohydrate (gm)

213.08 (94.19)

$251.13(111.62)$

$<$

0.001

Total sugars (gm)

$93.56(57.98)$

110.98 (68.26)

$<$

0.001

Total fat (gm)

$66.36(32.80)$

$76.58(39.85)$

$<$

0.001

Cholesterol (mg)

266.10 (175.92)

286.80 (197.44)

0.0102

Water (gm)

531.16 (584.86)

646.00 (647.87)

$<$

0.001

Physical Activity, Mean (SD)

Total activity (MET- $\quad 24.54(66.46)$

$57.68(98.97)$

$<$

h/week)

Abbreviations: $\mathrm{BMI}=$ body mass index $\mathrm{SBP}=$ systolic blood pressure, $\mathrm{DBP}=$ diastolic blood pressure, MET = Metabolic Equivalent of Task, SD = standard deviation 


\begin{tabular}{|c|c|c|c|}
\hline & $\begin{array}{l}\text { Congestive Heart Failure } \\
(n=660)\end{array}$ & $\begin{array}{l}\text { Non-Congestive Heart failure ( } \mathrm{n} \\
=20,923)\end{array}$ & $\begin{array}{l}P \\
\text { value }\end{array}$ \\
\hline $\begin{array}{l}\text { Vigorous activity (MET- } \\
\text { h/week) }\end{array}$ & $9.62(42.83)$ & $27.83(70.54)$ & $\begin{array}{l}< \\
0.001\end{array}$ \\
\hline $\begin{array}{l}\text { Moderate activity (MET- } \\
\text { h/week) }\end{array}$ & $11.89(28.80)$ & $23.64(42.33)$ & $<0.001$ \\
\hline $\begin{array}{l}\text { Sedimentary activity } \\
\text { (min/week) }\end{array}$ & $408.78(211.45)$ & $347.44(203.18)$ & $\begin{array}{l}< \\
0.001\end{array}$ \\
\hline $\begin{array}{l}\text { Walk or bicycle (MET- } \\
\text { h/week) }\end{array}$ & $3.03(14.95)$ & $6.21(20.27)$ & $<0.001$ \\
\hline \multicolumn{4}{|c|}{ Complete Blood Count - Whole Blood, Mean (SD) } \\
\hline Hemoglobin $(\mathrm{g} / \mathrm{dL})$ & $13.48(1.73)$ & $14.08(1.53)$ & $<0.001$ \\
\hline Hematocrit (\%) & $39.81(4.82)$ & $41.25(4.24)$ & $<.001$ \\
\hline
\end{tabular}

\section{Participants' characteristics by BMI category}

As shown in Table 2, the proportions of $\mathrm{BMI} \geq 30 \mathrm{~kg} / \mathrm{m} 2$ were $53.48 \%(\mathrm{n}=353)$ in CHF participants and $37.01 \%(n=7,744)$ in non-CHF participants. Compared with $\mathrm{BMI}<30 \mathrm{~kg} / \mathrm{m}^{2} \mathrm{CHF}$ participants, $\mathrm{BMI} \geq 30$ $\mathrm{kg} / \mathrm{m}^{2} \mathrm{CHF}$ participants showed significantly younger age, larger amount of water intake, longer sedentary time, and higher hematocrit (all $P<0.05$ ). Among non-CHF participants, those $\mathrm{BMI} \geq 30 \mathrm{~kg} / \mathrm{m}^{2}$ were older, had higher blood pressure and heart rate, lower energy, protein, total carbohydrate, and total sugar intakes but higher cholesterol and water intakes (all $P<0.05$ ). Physical activity level was lower and sedentary time was longer (both $P<0.05$ ) in $\mathrm{BMI} \geq 30 \mathrm{~kg} / \mathrm{m}^{2}$ non-CHF participants. Meanwhile, hemoglobin and hematocrit were lower in $\mathrm{BMI} \geq 30 \mathrm{~kg} / \mathrm{m}^{2}$ non-CHF participants than $\mathrm{BMI}<30 \mathrm{~kg} / \mathrm{m}^{2}$ ones (both $P<0.05$ ). 
Table 2

Characteristics of Congestive Heart Failure group and non-Congestive Heart Failure group (stratified by obese and non-obese)

\begin{tabular}{|c|c|c|c|c|c|c|c|}
\hline \multirow[t]{2}{*}{ BMI $\left(\mathrm{kg} / \mathrm{m}^{2}\right)$} & \multicolumn{3}{|c|}{ Congestive Heart Failure } & \multicolumn{3}{|c|}{ Non-Congestive Heart failure } & \multirow{2}{*}{$\begin{array}{l}\mathrm{P*} \\
\text { value }\end{array}$} \\
\hline & $\begin{array}{l}\mathrm{BMI}<30 \\
\mathrm{~kg} / \mathrm{m}^{2}(\mathrm{n}= \\
307)\end{array}$ & $\begin{array}{l}\mathrm{BMI} \geq 30 \\
\mathrm{~kg} / \mathrm{m}^{2}(\mathrm{n}= \\
353)\end{array}$ & $\begin{array}{l}\mathrm{P} \\
\text { value }\end{array}$ & $\begin{array}{l}\mathrm{BMI}<30 \\
\mathrm{~kg} / \mathrm{m}^{2}(\mathrm{n}= \\
13,179)\end{array}$ & $\begin{array}{l}\mathrm{BMI} \geq 30 \\
\mathrm{~kg} / \mathrm{m}^{2}(\mathrm{n}= \\
7,744)\end{array}$ & $\begin{array}{l}P \\
\text { value }\end{array}$ & \\
\hline \multicolumn{8}{|c|}{ Demographic and Weight Characteristics, Mean (SD) } \\
\hline Age (y) & $\begin{array}{l}68.67 \\
(12.86)\end{array}$ & $\begin{array}{l}64.54 \\
(12.30)\end{array}$ & 0.012 & $\begin{array}{l}48.28 \\
(18.17)\end{array}$ & $\begin{array}{l}49.29 \\
(16.30)\end{array}$ & $<.001$ & $<0.001$ \\
\hline \multicolumn{8}{|l|}{ Race } \\
\hline Male (No. ,\%) & $\begin{array}{l}178 \\
(57.98)\end{array}$ & $\begin{array}{l}181 \\
(51.27)\end{array}$ & $<.001$ & $\begin{array}{l}6,786 \\
(51.49)\end{array}$ & $\begin{array}{l}3,367 \\
(43.48)\end{array}$ & $<0.001$ & $<0.001$ \\
\hline BMI $\left(\mathrm{kg} / \mathrm{m}^{2}\right)$ & $\begin{array}{l}25.34 \\
(3.03)\end{array}$ & $\begin{array}{l}37.72 \\
(7.56)\end{array}$ & $\begin{array}{l}< \\
0.001\end{array}$ & $\begin{array}{l}24.95 \\
(3.13)\end{array}$ & $\begin{array}{l}35.86 \\
(5.78)\end{array}$ & $<0.001$ & $<0.001$ \\
\hline $\begin{array}{l}\text { SBP pressure } \\
(\mathrm{mmHg})\end{array}$ & $\begin{array}{l}130.38 \\
(23.38)\end{array}$ & $\begin{array}{l}129.34 \\
(20.63)\end{array}$ & 0.89 & $\begin{array}{l}122.01 \\
(18.53)\end{array}$ & $\begin{array}{l}125.40 \\
(17.33)\end{array}$ & $<001$ & $\begin{array}{l}< \\
0.001\end{array}$ \\
\hline $\mathrm{DBP}(\mathrm{mmHg})$ & $\begin{array}{l}64.13 \\
(14.56)\end{array}$ & $\begin{array}{l}66.81 \\
(15.33)\end{array}$ & 0.04 & $\begin{array}{l}69.15 \\
(12.45)\end{array}$ & $\begin{array}{l}71.51 \\
(13.16)\end{array}$ & $<.001$ & $<$ \\
\hline $\begin{array}{l}\text { Heart rate } \\
\text { (beats/min) }\end{array}$ & $\begin{array}{l}70.05 \\
(12.37)\end{array}$ & $\begin{array}{l}71.84 \\
(12.92)\end{array}$ & 0.24 & $\begin{array}{l}71.73 \\
(11.9)\end{array}$ & $\begin{array}{l}74.64 \\
(12.23)\end{array}$ & $<.001$ & $<0.001$ \\
\hline \multicolumn{8}{|c|}{ Total Nutrient Intakes, Mean (SD) } \\
\hline Energy (kcal) & $\begin{array}{l}1759.97 \\
(716.89)\end{array}$ & $\begin{array}{l}1712.74 \\
(718.01)\end{array}$ & 0.90 & $\begin{array}{l}2079.46 \\
(894.56)\end{array}$ & $\begin{array}{l}2003.43 \\
(832.49)\end{array}$ & $<.001$ & $\begin{array}{l}< \\
0.001\end{array}$ \\
\hline Protein (gm) & $\begin{array}{l}70.93 \\
(32.74)\end{array}$ & $\begin{array}{l}68.91 \\
(30.19)\end{array}$ & 0.90 & $\begin{array}{l}81.30 \\
(37.76)\end{array}$ & $\begin{array}{l}79.55 \\
(35.44)\end{array}$ & 0.005 & $<.001$ \\
\hline $\begin{array}{l}\text { Carbohydrate } \\
\text { (gm) }\end{array}$ & $\begin{array}{l}217.22 \\
(95.26)\end{array}$ & $\begin{array}{l}209.48 \\
(93.24)\end{array}$ & 0.81 & $\begin{array}{l}256.08 \\
(114.39)\end{array}$ & $\begin{array}{l}242.69 \\
(106.21)\end{array}$ & $<.001$ & $<0.001$ \\
\hline $\begin{array}{l}\text { Total sugars } \\
\text { (gm) }\end{array}$ & $\begin{array}{l}96.15 \\
(59.90)\end{array}$ & $\begin{array}{l}91.31 \\
(56.23)\end{array}$ & 0.80 & $\begin{array}{l}112.65 \\
(70.08)\end{array}$ & $\begin{array}{l}108.13 \\
(64.96)\end{array}$ & $<.001$ & $<0.001$ \\
\hline Total fat (gm) & $\begin{array}{l}65.65 \\
(30.90)\end{array}$ & $\begin{array}{l}66.98 \\
(34.41)\end{array}$ & 0.97 & $\begin{array}{l}76.48 \\
(40.51)\end{array}$ & $\begin{array}{l}76.73 \\
(38.72)\end{array}$ & 0.97 & $<0.001$ \\
\hline $\begin{array}{l}\text { Cholesterol } \\
(\mathrm{mg})\end{array}$ & $\begin{array}{l}261.71 \\
(181.87)\end{array}$ & $\begin{array}{l}269.92 \\
(170.75)\end{array}$ & 0.95 & $\begin{array}{l}282.87 \\
(200.12)\end{array}$ & $\begin{array}{l}293.50 \\
(192.62)\end{array}$ & $<.001$ & $\begin{array}{l}< \\
0.001\end{array}$ \\
\hline
\end{tabular}

Abbreviations: $\mathrm{BMI}=$ body mass index, $\mathrm{SBP}=$ systolic blood pressure, $\mathrm{DBP}=$ diastolic blood pressure, MET = Metaolic Equivalent of Task, SD = standard deviation

$P^{*}$ value was analysis between 4 groups 


\begin{tabular}{|c|c|c|c|c|c|c|c|}
\hline \multirow[t]{2}{*}{ BMI $\left(\mathrm{kg} / \mathrm{m}^{2}\right)$} & \multicolumn{3}{|c|}{ Congestive Heart Failure } & \multicolumn{3}{|c|}{ Non-Congestive Heart failure } & \multirow{2}{*}{$\begin{array}{l}\mathrm{P} * \\
\text { value }\end{array}$} \\
\hline & $\begin{array}{l}\mathrm{BMI}<30 \\
\mathrm{~kg} / \mathrm{m}^{2}(\mathrm{n}= \\
307)\end{array}$ & $\begin{array}{l}\mathrm{BMI} \geq 30 \\
\mathrm{~kg} / \mathrm{m}^{2}(\mathrm{n}= \\
353)\end{array}$ & $\begin{array}{l}P \\
\text { value }\end{array}$ & $\begin{array}{l}\mathrm{BMI}<30 \\
\mathrm{~kg} / \mathrm{m}^{2}(\mathrm{n}= \\
13,179)\end{array}$ & $\begin{array}{l}\mathrm{BMI} \geq 30 \\
\mathrm{~kg} / \mathrm{m}^{2}(\mathrm{n}= \\
7,744)\end{array}$ & $\begin{array}{l}P \\
\text { value }\end{array}$ & \\
\hline Water (gm) & $\begin{array}{l}457.69 \\
(484.71)\end{array}$ & $\begin{array}{l}595.06 \\
(653.72)\end{array}$ & 0.032 & $\begin{array}{l}615.04 \\
(621.65)\end{array}$ & $\begin{array}{l}698.70 \\
(687.04)\end{array}$ & $<.001$ & $\hat{0}_{0.001}$ \\
\hline \multicolumn{8}{|c|}{ Physical Activity, Mean (SD) } \\
\hline $\begin{array}{l}\text { Total activity } \\
\text { (MET-h/week) }\end{array}$ & $\begin{array}{l}28.17 \\
(67.00)\end{array}$ & $\begin{array}{l}21.38 \\
(65.91)\end{array}$ & 0.81 & $\begin{array}{l}60.85 \\
(101.41)\end{array}$ & $\begin{array}{l}52.28 \\
(94.43)\end{array}$ & $<.001$ & $\begin{array}{l}< \\
0.001\end{array}$ \\
\hline $\begin{array}{l}\text { Vigorous } \\
\text { activity (MET- } \\
\text { h/week) }\end{array}$ & $\begin{array}{l}11.36 \\
(41.45)\end{array}$ & $\begin{array}{l}8.11 \\
(43.99)\end{array}$ & 0.93 & $\begin{array}{l}29.53 \\
(71.50)\end{array}$ & $\begin{array}{l}24.93 \\
(68.79)\end{array}$ & $<.001$ & $\begin{array}{l}< \\
0.001\end{array}$ \\
\hline $\begin{array}{l}\text { Moderate } \\
\text { activity (MET- } \\
\text { h/week) }\end{array}$ & $\begin{array}{l}12.85 \\
(29.27)\end{array}$ & $\begin{array}{l}11.06 \\
(28.41)\end{array}$ & 0.94 & $\begin{array}{l}24.39 \\
(42.80)\end{array}$ & $\begin{array}{l}22.36 \\
(41.49)\end{array}$ & $<.001$ & $\begin{array}{l}<.001 \\
0 .\end{array}$ \\
\hline $\begin{array}{l}\text { Sedimentary } \\
\text { activity } \\
\text { (min/week) }\end{array}$ & $\begin{array}{l}379.29 \\
(195.98)\end{array}$ & $\begin{array}{l}434.43 \\
(221.13)\end{array}$ & 0.002 & $\begin{array}{l}337.23 \\
(199.05)\end{array}$ & $\begin{array}{l}364.81 \\
(208.91)\end{array}$ & $\begin{array}{l}< \\
0.001\end{array}$ & $\begin{array}{l}< \\
0.001\end{array}$ \\
\hline $\begin{array}{l}\text { Walk or bicycle } \\
\text { (MET-h/week) }\end{array}$ & $\begin{array}{l}3.97 \\
(16.42)\end{array}$ & $\begin{array}{l}2.21 \\
(13.51)\end{array}$ & 0.68 & $\begin{array}{l}6.93 \\
(21.51)\end{array}$ & $\begin{array}{l}4.99 \\
(17.92)\end{array}$ & $<.001$ & $\begin{array}{l}<.001 \\
0.00\end{array}$ \\
\hline \multicolumn{8}{|c|}{ Complete Blood Count - Whole Blood, Mean (SD) } \\
\hline $\begin{array}{l}\text { Hemoglobin } \\
(\mathrm{g} / \mathrm{dL})\end{array}$ & $\begin{array}{l}13.34 \\
(1.80)\end{array}$ & $\begin{array}{l}13.61 \\
(1.65)\end{array}$ & 0.121 & $\begin{array}{l}14.12 \\
(1.50)\end{array}$ & $\begin{array}{l}14.00 \\
(1.57)\end{array}$ & $\begin{array}{l}< \\
0.001\end{array}$ & $\begin{array}{l}< \\
0.001\end{array}$ \\
\hline Hematocrit (\%) & $\begin{array}{l}39.31 \\
(5.02)\end{array}$ & $\begin{array}{l}40.25 \\
(4.61)\end{array}$ & 0.028 & $\begin{array}{l}41.32 \\
(4.21)\end{array}$ & $\begin{array}{l}41.13 \\
(4.30)\end{array}$ & 0.009 & $<.001$ \\
\hline \multicolumn{8}{|c|}{$\begin{array}{l}\text { Abbreviations: BMI = body mass index, SBP = systolic blood pressure, } \mathrm{DBP}=\text { diastolic blood pressure, } \\
\mathrm{MET}=\text { Metaolic Equivalent of Task, SD = standard deviation }\end{array}$} \\
\hline$P$ value was a & (10 10 & sups & & & & & \\
\hline
\end{tabular}

\section{Discussion}

There are two major findings in the present study. First, compared with non-CHF participants, CHF participants exhibited higher BMI with lower energy and macronutrient intakes, lower physical activity level and longer rest time and hemodilution (lower hematocrit and hemoglobin). Second, higher water intake, sedentary time and hematocrit were observed in CHF patients with $\mathrm{BMI} \geq 30 \mathrm{~kg} / \mathrm{m}^{2} \mathrm{~s}$, while energy, macronutrient intake and physical level were similar between CHF patients with $\mathrm{BMI} \geq 30 \mathrm{~kg} / \mathrm{m}^{2}$ and < $30 \mathrm{~kg} / \mathrm{m}^{2}$. 
Excess body weight and a sedentary lifestyle are major public health problems worldwide [16]. Obesity $\left(\mathrm{BMI} \geq 30 \mathrm{~kg} / \mathrm{m}^{2}\right)$ has been considered as a major independent risk factor for cardiovascular disease [17]. A previous study showed that up to $40 \%$ of patients with CHF suffered from obesity [8]. The interplay between obesity and CHF is complex. Despite obesity increases the risk of CHF, studies found that $\mathrm{CHF}$ patients who developed obesity or over-weight $\left(25-29.9 \mathrm{~kg} / \mathrm{m}^{2}\right)$ are associated with better prognosis compared with those who did not [18-20], with reductions in cardiovascular disease mortality (19\% and $40 \%$, respectively) and all-cause mortality (16\% and $33 \%$, respectively) compared to heart failure patients with normal-weight (BMI $20-24.9 \mathrm{~kg} / \mathrm{m}^{2}$ ), whereas heart failure patients with $\mathrm{BMI}<20 \mathrm{~kg} / \mathrm{m}^{2}$ increased total and cardiovascular mortality (27\% and $20 \%$, respectively) [21]. This phenomenon is termed as "obesity paradox" and several potential reasons may account for it, such as earlier presentation, different etiology, greater metabolic reserves, protective cytokines, more tolerance of cardiac medications, different cardiorespiratory fitness level and less cachexia [22]. Based on previous studies, our study termed

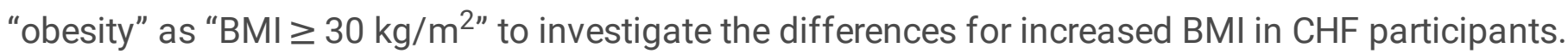

In our analysis, we recognized CHF participants presented with higher BMI than non-CHF participants and a higher proportion of $\mathrm{BMI} \geq 30 \mathrm{~kg} / \mathrm{m}^{2}$ among CHF participants. Meanwhile, we also noticed an inadequate energy and macronutrient intakes in CHF participants, seen as significantly lower energy, protein, carbohydrate, total sugar, total fat, cholesterol, and water intakes. This implied that nutritional deficiency existed in CHF participants. Malnutrition is commonly prevalent in $\mathrm{CHF}$, with a reported incidence of $7.5 \%$ and $57 \%$ in severe or moderate malnutrition, respectively [23]. These patients are older and frailty, with negative calorie and nitrogen balance and energy availability [24] for physical activity and are triggered by multiple factors such as anorexia, malabsorption secondary to intestinal edema, high energy demand, and cytokine-induced hypercatabolism [7, 25] and worse outcomes $[6,26,27]$. Although when compared with $\mathrm{CHF}$ patients with $\mathrm{BMI}<30 \mathrm{~kg} / \mathrm{m}^{2}$, those with $\mathrm{BMI} \geq 30 \mathrm{~kg} / \mathrm{m}^{2}$ participants did not show a distinct increase in energy and macronutrient intakes. A constellation of researches revealed that failing myocardium utilized glucose switching from fatty acids as the primary energetic substrate to produce ATP [28], which may exert a protective role in preventing cardiomyocytes from oxidative radical excess and cell damage [29, 30], but a lower energy production [31]. With more total fat intake and less glucose intake, cells may have the less energetic substrate to produce enough energy. This would affect metabolism in the body and cause adipose tissue accumulation, and subsequently increased body weight. This may imply that inadequate or unbalanced energy and macronutrient intakes are account for the increase in BMI or obesity among CHF participants. A daily caloric intake of about $29 \mathrm{kcal} / \mathrm{kg}$ and a daily protein intake of $1.2-1.4 \mathrm{~g} / \mathrm{kg}$ was recommended for $\mathrm{CHF}$ patients at normal weights and a less energy intake was required $(20-24 \mathrm{kcal} / \mathrm{kg} /$ day), and a reduction in dietary fat intake to about $25 \%$ of total caloric intake $(0.6-0.8 \mathrm{~g} / \mathrm{kg} / \mathrm{day})$ for overweight and obese CHF patients [32, 33].

Our study found lower hematocrit and hemoglobin levels in CHF participants, while CHF with BMI $\geq$ $30 \mathrm{~kg} / \mathrm{m}^{2}$ participants had higher hematocrit and hemoglobin concentration than those with $\mathrm{BMI}<$ $30 \mathrm{~kg} / \mathrm{m}^{2}$. These results demonstrated that hemodilution and fluid retention are common in $\mathrm{CHF}$, especially in those with lower BMI level $\left(\mathrm{BMI}<30 \mathrm{~kg} / \mathrm{m}^{2}\right)$. This observation may be another potential 
reason to explain "obesity paradox". Considerable evidence demonstrated that total blood volume and cardiac output are positively correlated with the degree of excess body weight $[18,22,34]$ and further preserved or even increased skeletal muscle mass (i.e. lean mass) [35, 36], which may cause BMI higher vice versa. This may explain the phenomenon of elevated hematocrit and hemoglobin concentration in $\mathrm{CHF}$ with $\mathrm{BMI} \geq 30 \mathrm{~kg} / \mathrm{m}^{2}$ participants and fluid retention may not be the reason for increased $\mathrm{BMI}$ or obesity in CHF participants. Hemodilution was common in chronic heart failure [37] and has a deleterious effect as it may impair peripheral oxygen delivery [38] and is often neglected as compensatory mechanisms may mask signs of volume [39]. Our study found lower hematocrit and hemoglobin level in CHF participants, while $\mathrm{CHF}$ with $\mathrm{BMI} \geq 30 \mathrm{~kg} / \mathrm{m}^{2}$ participants had higher hematocrit and hemoglobin concentration than $\mathrm{BMl}<30 \mathrm{~kg} / \mathrm{m}^{2}$ ones. These results demonstrated that hemodilution and fluid retention are common in CHF, especially in those with lower BMI level $\left(\mathrm{BMl}<30 \mathrm{~kg} / \mathrm{m}^{2}\right)$. Previous studies indicated a higher mortality rate in patients with hemodilution than in those with hemoconcentration in acute heart failure patients [15], while fluid restriction could only improve signs and symptoms of chronic heart failure in patients in moderate to severe chronic heart failure [40] and aggressive fluid removal positively affected survival [41]. In the present study, CHF with BMI $\geq 30 \mathrm{~kg} / \mathrm{m}^{2}$ participants had higher hematocrit and hemoglobin concentration than $\mathrm{BMl}<30 \mathrm{~kg} / \mathrm{m}^{2}$ ones, indicating that obese $\mathrm{CHF}$ participants were less likely to develop hemodilution. This could be one explanation for "obesity paradox". Furthermore, hemodynamic changes including increased stroke volume and increased arterial pressure may compensate the impaired peripheral oxygen delivery, leading CHF patients with increased $\mathrm{BMI}$ or obesity better prognoses. Still, we could not ignore that there was fluid retention in our CHF group which implied an inadequate usage of diuretics and fluid management should be enhanced.

Another finding in our analysis is that we demonstrated that CHF participants had lower physical activity levels and spent a longer time in rest, especially those $B M I \geq 30 \mathrm{~kg} / \mathrm{m}^{2}$ participants. It was revealed that sedentary time caused metabolic alterations at the muscle level and next influence gross metabolic disturbances in the whole body [42]. It has been reported that prolonged sedentary time would impair mitochondrial function by elevating oxidative stress levels [43], which decreased the mitochondrial respiration level [44] and caused insufficient ATP production for daily activity and metabolism. Emerging evidences have demonstrated a significant dose-response association between sitting time and cardiovascular disease mortality [45-48] and the relative risks associated with sedentary time were higher among participants without regular physical activity $[49,50]$. Meanwhile, there have been proven cardioprotective effects of regular physical activity on cardiovascular health, with improving cardiac compliance, reducing arterial stiffness and ventricular afterload, and finally reducing the risk of future cardiac dysfunction and improving cardiovascular outcomes [42]. Moreover, researches showed an increasing lean mass with resistance exercise training could effectively improve muscular fitness in CHF $[51,52]$, which could prevent sarcopenia [53] or even cachexia in CHF patients and have better prognosis [54].

Our investigation has several limitations. We only included macronutrient in our analysis and lacked the data of micronutrient intake. Therefore, the present study may have underestimated the nutritional deficit 
and its effects on obesity among participants. Also, details regarding the etiology, subtype (HF with reduced ejection fraction versus HF with preserved ejection fraction), and severity of HF were not available. Moreover, CHF participants included in our analysis were self-reported in the NHANES survey. This may lead to possible reporting and recall bias. Finally, we lacked follow-up data of these participants, including BMI changes and relative outcomes, so we were unable to recognize the effects of nutrients intake and physical activity on BMI changes and prognoses in patients with CHF.

\section{Conclusion}

Higher water intake, sedentary time and hematocrit were observed in $\mathrm{BMI} \geq 30 \mathrm{~kg} / \mathrm{m}^{2} \mathrm{CHF}$ participants. It seems that longer resting time and an unbalanced diet may be associated with a higher prevalence of increased BMI or obesity in CHF participants. Future research is warranted to explore the mechanisms underlying this finding and whether intentional weight loss with combination of diet, exercise and others could be contributing to better health outcomes in BMI $\geq 30 \mathrm{~kg} / \mathrm{m}^{2} \mathrm{CHF}$ participants.

\section{Declarations}

\section{Acknowledgements}

None

\section{Funding}

None

\section{Conflict of interest}

None

\section{Availability of data and materials}

Data that support the findings of this study are available NHANES, https://www.cdc.gov/nchs/nhanes/index.htm

\section{Authors' contributions}

TX: study concept and design; acquisition, analysis, or interpretation of data; drafting of the manuscript. HZ: study concept and design, acquisition, analysis, or interpretation of data; statistical analysis. ZM: acquisition, analysis, or interpretation of data; statistical analysis. HZ: acquisition, analysis, or interpretation of data; statistical analysis. YH: critical revision of the manuscript for important intellectual content; administrative, technical, or material support; study supervision QZ: critical revision of the manuscript for important intellectual content; administrative, technical, or material support; study supervision. DX: critical revision of the manuscript for important intellectual content; administrative, 
technical, or material support; study supervision. YZ: study concept and design; critical revision of the manuscript for important intellectual content; administrative, technical, or material support; study supervision. JZ: study concept and design; critical revision of the manuscript for important intellectual content; administrative, technical, or material support; study supervision. All authors read and approved the final manuscript.

Ethics approval and consent to participate

This study was reviewed and approved by the National Center for Health Statistics research ethics review board, and written informed consent was obtained from all NHANES participants.

IRB/ERB: Protocol \#2011-17

Consent for publication

Not applicable.

\section{References}

1. Ambrosy AP, Fonarow GC, Butler J, Chioncel O, Greene SJ, Vaduganathan M, Nodari S, Lam C, Sato N, Shah AN et al: The global health and economic burden of hospitalizations for heart failure: lessons learned from hospitalized heart failure registries. J AM COLL CARDIOL 2014, 63(12):1123-1133.

2. Ziaeian B, Fonarow GC: Epidemiology and aetiology of heart failure. NAT REV CARDIOL 2016, 13(6):368-378.

3. Lourenço BH, Vieira LP, Macedo A, Nakasato M, Marucci MF, Bocchi EA: Nutritional status and adequacy of energy and nutrient intakes among heart failure patients. ARQ BRAS CARDIOL 2009, 93(5):541-548.

4. Sciatti E, Lombardi C, Ravera A, Vizzardi E, Bonadei I, Carubelli V, Gorga E, Metra M: Nutritional Deficiency in Patients with Heart Failure. NUTRIENTS 2016, 8(7).

5. Makarewicz-Wujec M, Kozłowska-Wojciechowska M, Sygnowska E, Waśkiewicz A: Does heart failure determine the nutrition of patients? KARDIOL POL 2014, 72(1):56-63.

6. Chien SC, Lo Cl, Lin CF, Sung KT, Tsai JP, Huang WH, Yun CH, Hung TC, Lin JL, Liu CY et al: Malnutrition in acute heart failure with preserved ejection fraction: clinical correlates and prognostic implications. ESC Heart Fail 2019, 6(5):953-964.

7. Kinugawa S, Fukushima A: Malnutrition in Heart Failure: Important But Undervalued Issue. JACC Heart Fail 2018, 6(6):487-488.

8. Kapoor JR, Heidenreich PA: Obesity and survival in patients with heart failure and preserved systolic function: a U-shaped relationship. AM HEART J 2010, 159(1):75-80.

9. Hu G, Jousilahti P, Antikainen R, Katzmarzyk PT, Tuomilehto J: Joint effects of physical activity, body mass index, waist circumference, and waist-to-hip ratio on the risk of heart failure. CIRCULATION 
2010, 121(2):237-244.

10. Oreopoulos A, Padwal R, Kalantar-Zadeh K, Fonarow GC, Norris CM, McAlister FA: Body mass index and mortality in heart failure: a meta-analysis. AM HEART J 2008, 156(1):13-22.

11. Loprinzi PD: Physical activity, weight status, and mortality among congestive heart failure patients. INT J CARDIOL 2016, 214:92-94.

12. Milajerdi A, Djafarian K, Shab-Bidar S, Speakman JR: Pre- and post-diagnosis body mass index and heart failure mortality: a dose-response meta-analysis of observational studies reveals greater risk of being underweight than being overweight. OBES REV 2018, 20(2):252-261.

13. Zipf G, Chiappa M, Porter KS, Ostchega Y, Lewis BG, Dostal J: National health and nutrition examination survey: plan and operations, 1999-2010. Vital Health Stat 1 2013(56):1-37.

14. Executive summary of the clinical guidelines on the identification, evaluation, and treatment of overweight and obesity in adults. Arch Intern Med 1998, 158(17):1855-1867.

15. Zhou H, Xu T, Huang Y, Zhan Q, Huang X, Zeng Q, Xu D: The top tertile of hematocrit change during hospitalization is associated with lower risk of mortality in acute heart failure patients. $B M C$ Cardiovasc Disord 2017, 17(1):235.

16. Ng M, Fleming T, Robinson M, Thomson B, Graetz N, Margono C, Mullany EC, Biryukov S, Abbafati C, Abera SF et al: Global, regional, and national prevalence of overweight and obesity in children and adults during 1980-2013: a systematic analysis for the Global Burden of Disease Study 2013. LANCET 2014, 384(9945):766-781.

17. Lavie CJ, De Schutter A, Parto P, Jahangir E, Kokkinos P, Ortega FB, Arena R, Milani RV: Obesity and Prevalence of Cardiovascular Diseases and Prognosis-The Obesity Paradox Updated. PROG CARDIOVASC DIS 2016, 58(5):537-547.

18. Lavie CJ, Alpert MA, Arena R, Mehra MR, Milani RV, Ventura HO: Impact of obesity and the obesity paradox on prevalence and prognosis in heart failure. JACC Heart Fail 2013, 1(2):93-102.

19. Futter JE, Cleland JG, Clark AL: Body mass indices and outcome in patients with chronic heart failure. EUR J HEART FAIL 2011, 13(2):207-213.

20. Bozkurt $\mathrm{B}$, Deswal $\mathrm{A}$ : Obesity as a prognostic factor in chronic symptomatic heart failure. $A M H E A R T$ $J$ 2005, 150(6):1233-1239.

21. Sharma A, Lavie CJ, Borer JS, Vallakati A, Goel S, Lopez-Jimenez F, Arbab-Zadeh A, Mukherjee D, Lazar JM: Meta-analysis of the relation of body mass index to all-cause and cardiovascular mortality and hospitalization in patients with chronic heart failure. AM J CARDIOL 2015, 115(10):1428-1434.

22. Parto $P$, Lavie $C J$, Arena R, Bond $S$, Popovic D, Ventura HO: Body habitus in heart failure: understanding the mechanisms and clinical significance of the obesity paradox. Future Cardio/2016, 12(6):639-653.

23. Sze S, Pellicori P, Zhang J, Clark AL: Malnutrition, congestion and mortality in ambulatory patients with heart failure. HEART 2019, 105(4):297-306. 
24. Sanches MDK, Dalira SPI, Clausell N, Corrêa SG: Adequacy of energy and nutrient intake in patients with heart failure. Nutr Hosp 2015, 31(1):500-507.

25. Aquilani R, Opasich $\mathrm{C}$, Verri $\mathrm{M}$, Boschi $\mathrm{F}$, Febo $\mathrm{O}$, Pasini $\mathrm{E}$, Pastoris $\mathrm{O}$ : Is nutritional intake adequate in chronic heart failure patients? J AM COLL CARDIOL 2003, 42(7):1218-1223.

26. Maeda D, Kanzaki Y, Sakane K, Ito T, Sohmiya K, Hoshiga M: Prognostic impact of a novel index of nutrition and inflammation for patients with acute decompensated heart failure. HEART VESSELS 2020.

27. Nakamura T, Matsumoto M, Haraguchi $Y$, Ishida T, Momomura SI: Prognostic impact of malnutrition assessed using geriatric nutritional risk index in patients aged $\geqslant 80$ years with heart failure. Eur $J$ Cardiovasc Nurs 2020, 19(2):172-177.

28. Taegtmeyer H, Sen S, Vela D: Return to the fetal gene program: a suggested metabolic link to gene expression in the heart. Ann N Y Acad Sci 2010, 1188:191-198.

29. Park TS, Goldberg IJ: Sphingolipids, lipotoxic cardiomyopathy, and cardiac failure. HEART FAIL CLIN 2012, 8(4):633-641.

30. Libby $P$, Maroko PR, Braunwald $E$ : The effect of hypoglycemia on myocardial ischemic injury during acute experimental coronary artery occlusion. CIRCULATION 1975, 51(4):621-626.

31. Neubauer S: The failing heart-an engine out of fuel. N Engl J Med 2007, 356(11):1140-1151.

32. Kopple JD: National kidney foundation K/DOQI clinical practice guidelines for nutrition in chronic renal failure. AM J KIDNEY DIS 2001, 37(1 Suppl 2):S66-S70.

33. Arkinstall MJ, Bruce CR, Clark SA, Rickards CA, Burke LM, Hawley JA: Regulation of fuel metabolism by preexercise muscle glycogen content and exercise intensity. J Appl Physiol (1985) 2004, 97(6):2275-2283.

34. Alpert MA: Obesity cardiomyopathy: pathophysiology and evolution of the clinical syndrome. $A M J$ MED SCI 2001, 321(4):225-236.

35. Collis T, Devereux RB, Roman MJ, de Simone G, Yeh J, Howard BV, Fabsitz RR, Welty TK: Relations of stroke volume and cardiac output to body composition: the strong heart study. CIRCULATION2001, 103(6):820-825.

36. Carbone S, Lavie CJ, Arena R: Obesity and Heart Failure: Focus on the Obesity Paradox. MAYO CLIN PROC 2017, 92(2):266-279.

37. Androne AS, Katz SD, Lund L, LaManca J, Hudaihed A, Hryniewicz K, Mancini DM: Hemodilution is common in patients with advanced heart failure. CIRCULATION 2003, 107(2):226-229.

38. Chapler $\mathrm{CK}$, Cain SM: The physiologic reserve in oxygen carrying capacity: studies in experimental hemodilution. Can J Physiol Pharmacol 1986, 64(1):7-12.

39. Wilson JR, Lanoce V, Frey MJ, Ferraro N: Effect on peripheral arterioles of chronic fluid and sodium retention in heart failure. J AM COLL CARDIOL 1988, 12(1):202-208.

40. Philipson H, Ekman I, Forslund HB, Swedberg K, Schaufelberger M: Salt and fluid restriction is effective in patients with chronic heart failure. EUR J HEART FAIL 2013, 15(11):1304-1310. 
41. Testani JM, Chen J, McCauley BD, Kimmel SE, Shannon RP: Potential effects of aggressive decongestion during the treatment of decompensated heart failure on renal function and survival. CIRCULATION2010, 122(3):265-272.

42. Lavie CJ, Ozemek C, Carbone S, Katzmarzyk PT, Blair SN: Sedentary Behavior, Exercise, and Cardiovascular Health. CIRC RES 2019, 124(5):799-815.

43. Joseph AM, Adhihetty PJ, Buford TW, Wohlgemuth SE, Lees HA, Nguyen LM, Aranda JM, Sandesara $\mathrm{BD}$, Pahor $\mathrm{M}$, Manini TM et al: The impact of aging on mitochondrial function and biogenesis pathways in skeletal muscle of sedentary high- and low-functioning elderly individuals. AGING CELL 2012, 11(5):801-809.

44. Conley KE, Amara CE, Bajpeyi S, Costford SR, Murray K, Jubrias SA, Arakaki L, Marcinek DJ, Smith SR: Higher mitochondrial respiration and uncoupling with reduced electron transport chain content in vivo in muscle of sedentary versus active subjects. J Clin Endocrinol Metab 2013, 98(1):129-136.

45. Young DR, Hivert MF, Alhassan S, Camhi SM, Ferguson JF, Katzmarzyk PT, Lewis CE, Owen N, Perry CK, Siddique J et al: Sedentary Behavior and Cardiovascular Morbidity and Mortality: A Science Advisory From the American Heart Association. CIRCULATION 2016, 134(13):e262-e279.

46. Katzmarzyk PT, Church TS, Craig CL, Bouchard C: Sitting time and mortality from all causes, cardiovascular disease, and cancer. Med Sci Sports Exerc 2009, 41(5):998-1005.

47. Chau JY, Grunseit AC, Chey T, Stamatakis E, Brown WJ, Matthews CE, Bauman AE, van der Ploeg HP: Daily sitting time and all-cause mortality: a meta-analysis. PLOS ONE 2013, 8(11):e80000.

48. Pandey A, Salahuddin U, Garg S, Ayers C, Kulinski J, Anand V, Mayo H, Kumbhani DJ, de Lemos J, Berry JD: Continuous Dose-Response Association Between Sedentary Time and Risk for Cardiovascular Disease: A Meta-analysis. JAMA CARDIOL 2016, 1(5):575-583.

49. Biswas A, Oh PI, Faulkner GE, Bajaj RR, Silver MA, Mitchell MS, Alter DA: Sedentary time and its association with risk for disease incidence, mortality, and hospitalization in adults: a systematic review and meta-analysis. ANN INTERN MED 2015, 162(2):123-132.

50. Ekelund U, Steene-Johannessen J, Brown WJ, Fagerland MW, Owen N, Powell KE, Bauman A, Lee IM: Does physical activity attenuate, or even eliminate, the detrimental association of sitting time with mortality? A harmonised meta-analysis of data from more than 1 million men and women. LANCET 2016, 388(10051):1302-1310.

51. Lavie CJ, Forman DE, Arena R: Bulking Up Skeletal Muscle to Improve Heart Failure Prognosis. JACC Heart Fail 2016, 4(4):274-276.

52. Ventura HO, Carbone S, Lavie $\mathrm{CJ}$ : Muscling up to improve heart failure prognosis. EUR J HEART FAIL 2018, 20(11):1588-1590.

53. Prado CM, Heymsfield SB: Lean tissue imaging: a new era for nutritional assessment and intervention. JPEN J Parenter Enteral Nutr 2014, 38(8):940-953.

54. Bekfani T, Pellicori P, Morris DA, Ebner N, Valentova M, Steinbeck L, Wachter R, Elsner S, Sliziuk V, Schefold JC et al: Sarcopenia in patients with heart failure with preserved ejection fraction: Impact on muscle strength, exercise capacity and quality of life. INT J CARDIOL 2016, 222:41-46. 
Figures

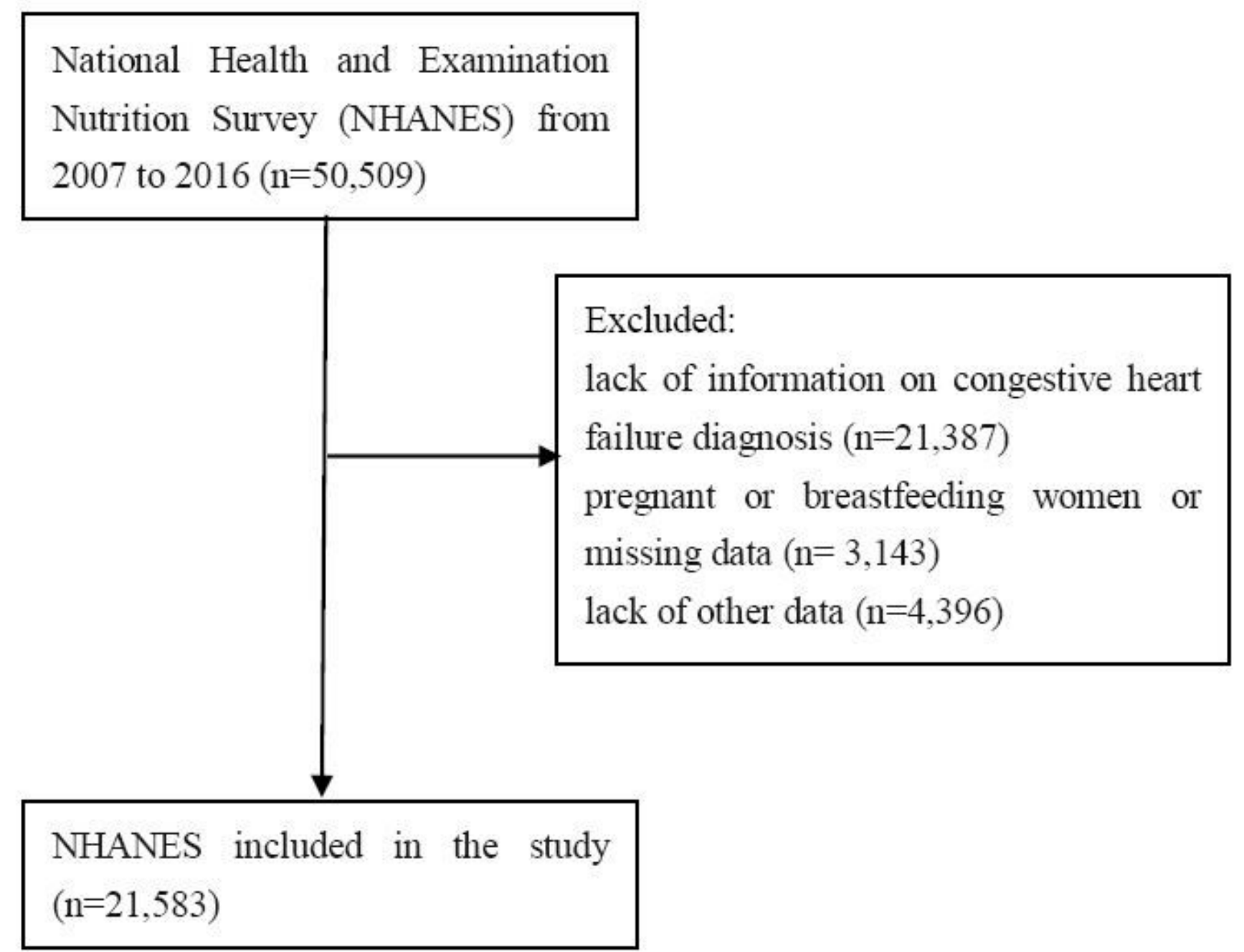

Figure 1

Flow chart for participant selection 